\title{
EGFR Mutation Status and TKI Therapy in Patients with Advanced Vulvar Cancer: Clinical Observations
}

\author{
Kun Liu1, Yong Yang2*, Cunde Wang ${ }^{3}$, Yufeng Wang1, Ying Zhu' ${ }^{1}$ \\ ${ }^{1}$ Department of Cadres Medical, The Affiliated Tumor Hospital of Kunming Medical University, Kunming, China \\ ${ }^{2}$ Department of Urology, The Affiliated Tumor Hospital of Kunming Medical University, Kunming, China \\ ${ }^{3}$ Research Center of Tumor Palliative Medicine in Yunnan, Kunming, China \\ Email: *lkyy_1975@163.com
}

How to cite this paper: Liu, K., Yang, Y., Wang, C.D., Wang, Y.F. and Zhu, Y. (2017) EGFR Mutation Status and TKI Therapy in Patients with Advanced Vulvar Cancer: Clinical Observations. Open Access Library Journal, 4: e3956.

https://doi.org/10.4236/oalib.1103956

Received: September 20, 2017

Accepted: October 17, 2017

Published: October 20, 2017

Copyright $\odot 2017$ by authors and Open Access Library Inc.

This work is licensed under the Creative Commons Attribution International License (CC BY 4.0).

http://creativecommons.org/licenses/by/4.0/

\begin{abstract}
Vulvar cancer is a rare female genital neoplasm representing $5 \%$ of all gynecological malignancies, and occurring most frequently in women between the ages of 65 and 75. The most common histological type is squamous cell carcinoma followed by melanoma, basal cell carcinoma, and adenocarcinoma. In the early disease stage, surgical treatment can be effective; however, once recurrence and metastasis occur, advanced vulvar cancer is often difficult to be treated. Overexpression, amplification, and mutations in epidermal growth factor receptor (EGFR) have been found in many types of cancer, including primary vulvar squamous cell carcinoma and metastatic lesions, and correlate with a poor prognosis. EGFR mutation detection has become a routine molecular test with significant implications for prognosis. Thus, the goal of this study was to learn more about EGFR gene somatic mutations in vulvar cancer and to determine whether EGFR-tyrosine kinase inhibitors (TKIs) have different efficacies in patients with and without EGFR mutations. The results showed that targeted therapy based on EGFR mutation status could significantly improve the prognosis of patients. Thus, molecular profiling to determine mutation status may be an initial step towards developing effective therapeutic regimens to treat advanced vulvar cancer.
\end{abstract}

\section{Subject Areas}

Oncology

\section{Keywords}

Gefitinib, Prognosis, Response Rate, Survival 


\section{Introduction}

Vulvar cancer is the fourth most common gynecologic cancer in the United States, accounting for $5 \%$ of cancers of the female genital tract and $1 \%$ of all female cancers [1]. This disease occurs most frequently in women between the ages of 65 and 75 [2] [3]. However, the rate of vulvar cancers diagnosed in younger women has been increasing in the last decade, as a result of being caused by human papilloma virus (HPV) infection [4]. These HPV-associated vulvar cancers are often seen in women under 45 years of age. Other risk factors for vulvar cancer include vulvar intraepithelial neoplasia, heavy cigarette smoking, lichen sclerosus, squamous hyperplasia, squamous carcinoma of the vagina or cervix, and chronic granulomatous diseases [1].

About $90 \%$ of vulvar cancers are squamous cell carcinomas and about $5 \%$ are melanomas. The classic symptom is vulvar itching (pruritus), which is reported in almost $90 \%$ of women with vulvar cancer. There can also be associated pain, pain with intercourse, bleeding, vaginal discharge, and/or painful urination (dysuria). Pre-cancerous lesions or early vulvar cancers may have mild or minimal symptoms, and preventive gynecologic exams can be helpful in detecting these early lesions. Ultimately, many women will develop a visible vulvar mass: the squamous cell subtype can look like elevated white, pink, or red bumps; whereas vulvar melanoma characteristically presents as a colored, ulcerated growth [1]. Survival rates tend to be better in younger women as they often present in the early stages of the disease. On the other hand, older women are less likely to attend preventive gynecologic healthcare visits, and as such, are more likely to be diagnosed with advanced vulvar cancer. Higher mortality rates and poorer survival are associated with more aggressive disease, thus the burden of vulvar cancer mainly lies with older women [5].

Vulvar cancer can spread by growing into adjacent areas such as the vagina and anus. Surgery, radiation therapy, and chemotherapy are all treatment options that are typically used in various combinations. In the early disease stage, surgical treatment is dominant; however, once recurrence and metastasis occur, advanced vulvar cancer is often difficult to be treated. In addition, chemotherapy has low efficiency and high toxicity, resulting in a 5-year survival rate of less than $5 \%$ in patients with advanced vulvar cancer. Thus, it is clear that new therapeutic strategies are needed.

The development of cancer genomics has greatly promoted individualized tumor therapies and enabled the development of molecular targeted cancer therapy, which is a revolutionary approach to treating cancer. Epidermal growth factor receptor (EGFR) is a protein tyrosine kinase cell-surface receptor for members of the EGF family of extracellular protein ligands. Overexpression, amplification, and mutations in EGFR have been found in many types of cancer, including vulvar cancer, and are linked with a poor prognosis [6]. Small-molecule EGFR tyrosine kinase inhibitors (EGFR-TKIs) have been successfully used in the clinical treatment of several types of cancers. For example, erlotinib (Tarceva) was 
the first EGFR-TKI used to treat vulvar cancer [7]; the treatment was well tolerated with no serious side effects, and continues to be one of the active agents available to treat this disease [1] [8].

Although erlotnib has shown some efficacy in treating vulvar cancer, metastatic vulvar cancer remains difficult to treat with poor clinical outcomes [8] [9] [10]. Thus, the goal of this study was to learn more about EGFR gene somatic mutations in vulvar cancer and to determine whether EGFR-TKIs have different efficacies in patients with and without EGFR mutations. To this end, 30 patients with advanced vulvar cancer, who were admitted to our hospital (The Affiliated Tumor Hospital of Kunming Medical University) between January 2008 and October 2015, were treated with the small-molecule EGFR-TKI gefitinib (Irresa). EGFR genetic testing was performed, and seven cases with EGFR E19/21 mutations and two cases with EGFR E20 mutations were found. The clinical efficacy of gefitinib in all of the patients was summarized and analyzed. The results showed that targeted therapy based on EGFR mutation status could significantly improve the prognosis of patients. Thus, using molecular profiling to determine mutation status may be an initial step towards developing effective therapeutic regimens to treat advanced vulvar cancer.

\section{Materials and Methods}

\subsection{General Information}

Between January 2008 and October 2015, 30 patients with diagnosed advanced vulvar cancer who were admitted to our hospital (The Affiliated Tumor Hospital of Kunming Medical University), were included in this study, Patient age ranged from 36 to 75 years, with a median age of 68 years. With regard to patient characteristics, 27 women were post-menopausal and on average had three gestations and two births; 26 patients (86.67\%) had squamous cell carcinoma, 3 (10\%) had adenocarcinoma, and 1 patient (3.33\%) had basal cell carcinoma. The patient subsets included those with recurrence after chemoradiotherapy and surgery, occurrence of distant metastases with advanced vulvar cancer, newly diagnosed with stage IV vulvar cancer, disease progression despite treatment with platinum chemotherapy/chemotherapy concomitant with radiotherapy, and those who rejected radiochemotherapy treatment.

\subsection{Treatment}

All of the patients took a routine blood test before being given oral gefitinib. Their liver and kidney function and electrocardiogram (ECG) were all in the normal range. They were administered oral gefitinib, $250 \mathrm{mg}$ once daily, taken with or without food. Gefitinib administration and observation were continued until disease progression; treatment was terminated if intolerable severe toxicity occurred. Toxic side effects during treatment, progression-free survival (PFS), and overall survival (OS) were monitored. 


\subsection{Sample Collection and Detection}

Genomic DNA in paraffin-embedded tumor sections from the 30 patients was extracted using Maxwell ${ }^{\circ}$ system (Promega, Atlanta, GA, USA). DNA concentrations were detected with a spectrophotometer (Nanodrop 1000 spectrophotometer, Thermo Scientific, Waltham, MA, USA). The amount of extracted DNA samples was sufficient for detection of mutations. EGFR 19/20/21 and KRAS E2 mutations were detected by xTAG liquid chip technology. The detection methodology included five main steps: 1) Multiplex PCR amplification of specific regions of the target gene; 2) Treatment of the PCR reaction with Exonuclease I and shrimp alkaline phosphatase (EXO-SAP) to remove excess nucleotides and primers; 3) PCR product processing using allele specific primer extension (ASPE). In this reaction, each specific primer pair is complementary to a specific gene fragment. Each primer pair is connected with a universal label. Tsp DNA Polymerase only extends when the primers and template exactly match; 4) Hybridization with microspheres, in which tagged probes were wrapped in polystyrene microspheres and ASPE products were hybridized with specific probes; 5) Luminex assay using the Luminex 200 system followed by analysis of the median fluorescence intensity (MFI).

\subsection{Outcome Measures}

Efficacy was evaluated according to Response Evaluation Criteria In Solid Tumors (RECIST) 1.1 criteria.

Remarks-Evaluation of target lesions

Complete Response (CR): Disappearance of all target lesions. Any pathological lymph nodes (whether target or non-target) must have reduction in short axis to $<10 \mathrm{~mm}$.

Partial Response (PR): At least a 30\% decrease in the sum of diameters of target lesions, taking as reference the baseline sum diameters.

Progressive Disease (PD): At least a 20\% increase in the sum of diameters of target lesions, taking as reference the smallest sum on study (this includes the baseline sum if that is the smallest on study). In addition to the relative increase of $20 \%$, the sum must also demonstrate an absolute increase of at least $5 \mathrm{~mm}$. (Note: the appearance of one or more new lesions is also considered progression).

Stable Disease (SD): Neither sufficient shrinkage to qualify for PR nor sufficient increase to qualify for PD, taking as reference the smallest sum diameters while on study.

Patients were divided into those with complete remission (CR), partial response (PR), stable disease (SD), and progressive disease (PD). CR + PR calculated efficiency, and $\mathrm{CR}+\mathrm{PR}+\mathrm{SD}$ calculated disease control rate. PFS was defined as the time from the start of gefitinib treatment to disease progression. Overall survival (OS) was defined as time from the start of gefitinib treatment to death caused by any reason. Secondary outcome measures included EGFR gene muta- 
tions and observation of gefitinib efficacy.

\subsection{Follow-Up}

Patients were followed up monthly for efficacy assessment in the first 3 months after treatment with gefitinib, and subsequently assessed once every two months. Follow-up deadline is October 31, 2015. The main assessments were pelvic magnetic resonance imaging (MRI) or computed tomography (CT), abdominal ultrasound, analysis of liver and kidney functions, blood test and other tests.

\subsection{Statistical Analysis}

The SPSS v15.0 statistical software was used for analysis. The $\chi^{2}$ test was used to compare sample rates of EGFR E19/E21 mutations. The exact test was used to detect group differences using frequency (count) data, and response rate (RR) and DCR were compared between patient groups after treatment with gefitinib. Kaplan-Meier analysis was used to analyze the PFS between patient groups. $P<$ 0.05 was considered statistically significant. As for a retrospective study, explained how indeterminate results, missing data, and data outliers were handled by multiple imputation.

\section{Results}

\subsection{Relationship between EGFR Mutations with Gefitinib Efficacy}

Thirty patients with advanced vulvar cancer were treated with gefitinib after giving signed informed consent. EGFR mutations were detected in the surgical or biopsy specimens. The mutation rate was 30.0\% (9/30), and EGFR wild-type patients accounted for $70 \%$ (21/30) of the patient population. Assessment of treatment results of gefitinib in advanced vulvar cancer patients showed that the efficacy rate was $44.5 \%$ (4/9) in patients with EGFR mutations and the DCR was $66.7 \%(6 / 9)$. The objective response rate (ORR) was $14.3 \%(3 / 21)$ in patients with wild-type EGFR and the CR was $14.3 \%(3 / 21)$. Treatment efficacy was better in patients with EGFR mutants than with wild-type EGFR. Statistical analysis of treatment efficacy in the two groups showed that the difference in DCR was significant $(P<0.05$, Table 1$)$, suggesting that EGFR gene mutation status in patients with advanced vulvar cancer may guide targeted drug therapy in clinical.

Table 1. Comparison of RR and DCR between patients with EGFR mutations and wild-type (\%).

\begin{tabular}{cccc}
\hline Group & $\mathbf{n}$ & Response rate & Disease control rate (DCR) \\
\hline EGFR (+) & 9 & $4(44.5)$ & $6(66.7)$ \\
EGFR (-) & 21 & $3(14.3)$ & $3(14.3)$ \\
$\chi^{2}$ & & 3.203 & 4.983 \\
$\mathbf{P}$ & 0.096 & 0.035 \\
\hline
\end{tabular}




\subsection{Evaluation}

None of the patients had complete remission (complete response, CR), 7 (23.3\%) had partial remission/partial response (PR), 2 (6.7\%) had SD, and $21(70.0 \%)$ patients had PD. The ORR was $23.3 \%$ and the DCR was $30.0 \%$.

\subsection{Prognosis}

According to follow-up, the median PFS in patients with EGFR gene mutations was 108 days, the 1 -year survival rate was $33.3 \%$ (3/9), and the 2 -year survival rate was $11.1 \%(1 / 9)$. The median PFS in patients with wild-type EGFR gene was 49 days, the 1-year survival rate was $9.5 \%$ (2/21), and the 2 -year survival rate was $4.7 \%(1 / 21)$. The difference in survival time between the two groups was statistically significant ( $P=0.42$, Figure 1 ). Thus, clinical targeted drug therapy guided by EGFR mutation status may significantly improve the prognosis of patients.

\subsection{Adverse Reactions of Gefitinib Treatment}

Adverse reactions of gefitinib are mainly rash, diarrhea, elevated transaminases, nausea, vomiting, leukopenia, and interstitial lung disease. In this patient group, the most common reaction was an acne-like rash, which had an occurrence rate of $36.67 \%$ (11/30), followed by pruritus at a rate of $13.33 \%$ (4/30). Antihistamine treatment alleviated these symptoms. Patients did not experience diarrhea, elevated transaminase levels, nausea and vomiting, leukopenia or interstitial lung disease, or other severe adverse reactions. No patient reduced the dose or stopped taking the drug due to side effects.

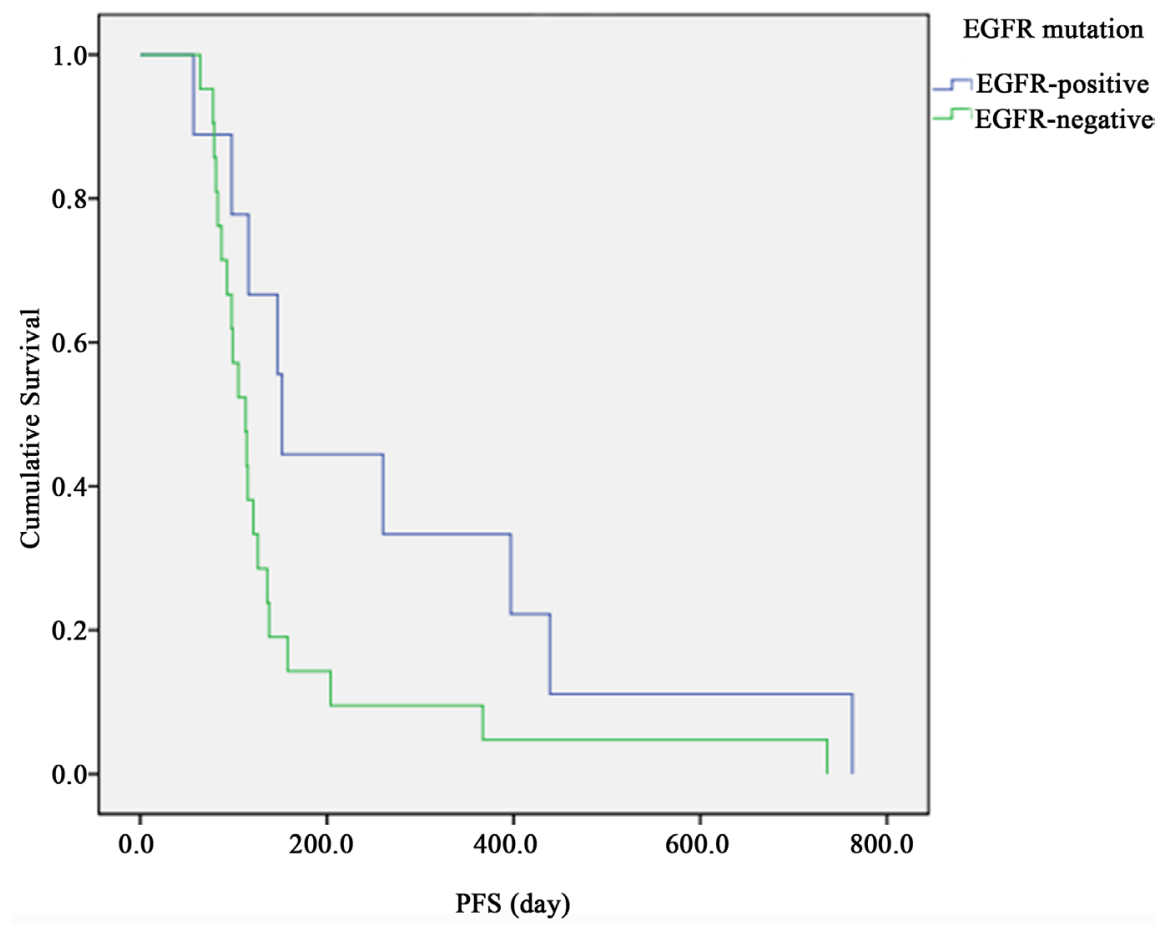

Figure 1. Analysis of Kaplan-Meier OS curves in the two patient groups. 


\section{Discussion}

Vulvar cancer results from abnormal cells growing on the surface of the vulvar skin. The prognosis for this disease is best when treated in the early stage, but it often does not cause early signs or symptoms. Thus, many cases are not discovered until the advanced stage [11]. Comprehensive treatments such as chemotherapy and surgical treatment result in a 5 -year survival rate of about 53\% $72 \%$ [12], and the recurrence rate of squamous cell carcinoma of the vulva is high. In the event of recurrence, chemotherapy has low efficacy and great side effects; thus, traditional treatments are not effective in controlling the progress of this disease.

In recent years, there has been considerable progress in the development of individualized drug therapy based on molecular markers and gene mutation detection [13]. Small-molecule EGFR-TKI is one of the target molecules in anti-cancer drug research field that has gained much attention. It works mainly by competitively and specifically binding the ATP binding site of the EGFR kinase domain, thereby inhibiting its kinase activity, resulting in inhibition of cancer cell proliferation and metastasis. Compared to traditional chemotherapy drugs, targeted drugs overcome the general common side effects of chemotherapy drugs, such as myelosuppression and gastrointestinal reactions. Representative EGFR-TKIs are erlotinib and gefitinib, which are widely used in the treatment of patients with non-small cell lung cancer. EGFR gene mutation status is an effective predictor of response to treatment [14]. Erlotinib (Teluo Kai) was the first EGFR-TKI used to treat advanced vulvar cancer [8] [15]. In addition, the combination of another EGFR-TKI, cetuximab, with a platinum formulation has been shown to have treatment efficacy and improve survival time in patients with advanced metastatic vulvar cancer [16]. However, metastatic vulvar cancer remains difficult to treat with poor clinical outcomes.

The goal of this study was to evaluate the relationship between EGFR mutation rate and treatment efficacy of gefitinib in patients with vulvar cancer. In our study group of 30 patients with advanced vulvar cancer, the mutation rate was $30.0 \%$ (9/30); EGFR wild-type patients accounted for 70\% (21/30) of the group. Assessment of gefitinib treatment showed that the effective rate in patients with EGFR mutations was $44.5 \%$ (4/9) and the DCR was $66.7 \%$ (6/9). The effective rate in patients with EGFR wild-type was $14.3 \%(3 / 21)$ and the DCR was $14.3 \%$ $(3 / 21)$. Thus, the DCR was more effective in patients with EGFR mutations compared to those with EGFR wild-type, suggesting that gefitinib may significantly improve the prognosis of patients with EGFR mutations. The most common side effect was an acne-like rash, followed by itching; however, all symptoms were mild and were alleviated with antihistamine treatment.

The limitation to this study is the fact that the number of patients enrolled in the study group was small. In addition, different patient factors such as race, age, ethnic group, and pathological disease type may cause different responses to treatment. In fact, studies have shown that younger women and elderly women have 
different types of squamous cell vulvar cancer, which behave differently and respond differently to treatment. Thus, further studies are still needed.

\section{Conclusion}

In summary, the results of this study show that EGFR gene mutation tests from vulvar tissues may provide guidance for targeted therapy in patients with advanced vulvar cancer, thereby improving prognosis and providing a new therapeutic approach for treating this disease.

\section{Recommendations}

Targeted therapy based on EGFR mutation status might improve prognosis in patients with advanced vulvar cancer, molecular profiling to determine mutation status may be an initial step towards developing effective therapeutic regimens to treat advanced vulvar cancer.

\section{References}

[1] Inrhaoun, H., Elghissassi, I., Gutierrez, M., Brain, E. and Errihani, H. (2012) Long Term Response to Erlotinib in a Patient with Recurrent Vulvar Carcinoma: Case Report and Review of Literature. Gynecologic Oncology Case Reports, 2, 119-120. https://doi.org/10.1016/j.gynor.2012.07.002

[2] Hampl, M., Deckers-Figiel, S., Hampl, J.A., Rein, D. and Bender, H.G. (2008) New Aspects of Vulvar Cancer: Changes in Localization and Age of Onset. Gynecologic Oncology, 109, 340-345. https://doi.org/10.1016/j.ygyno.2008.01.041

[3] Siegel, R., Naishadham, D. and Jemal, A. (2012) Cancer Statistics, 2012. CA: $A$ Cancer Journal for Clinicians, 62, 10-29. https://doi.org/10.3322/caac.20138

[4] Smith, J.S., Backes, D.M., Hoots, B.E., Kurman, R.J. and Pimenta, J.M. (2009) Human Papillomavirus Type-Distribution in Vulvar and Vaginal Cancers and Their Associated Precursors. Obstetrics \& Gynecology, 113, 917-924. https://doi.org/10.1097/AOG.0b013e31819bd6e0

[5] Stuckey, A., Schutzer, M., Rizack, T. and Dizon, D. (2013) Locally Advanced Vulvar Cancer in Elderly Women: Is Chemoradiation Beneficial? American Journal of Clinical Oncology, 36, 279-282. https://doi.org/10.1097/COC.0b013e3182467e9f

[6] Woelber, L., Hess, S., Bohlken, H., Tennstedt, P., Eulenburg, C., Simon, R., et al. (2012) EGFR Gene Copy Number Increase in Vulvar Carcinomas Is Linked with Poor Clinical Outcome. Journal of Clinical Pathology, 65, 133-139. https://doi.org/10.1136/jcp-2010-079806

[7] Olawaiye, A., Lee, L.M., Krasner, C. and Horowitz, N. (2007) Treatment of Squamous Cell Vulvar Cancer with the Anti-EGFR Tyrosine Kinase Inhibitor Tarceva. Gynecologic Oncology, 106, 628-630. https://doi.org/10.1016/j.ygyno.2007.05.006

[8] Horowitz, N., Olawaiye, A., Borger, D., Growdon, W., Krasner, C., Matulonis, U., et al. (2012) Phase II Trial of Erlotinib in Women with Squamous Cell Carcinoma of the Vulva. Gynecologic Oncology, 127, 141-146. https://doi.org/10.1016/j.ygyno.2012.06.028

[9] Fu, S., Shi, N., Wheler, J., Naing, A., Janku, F., Piha-Paul, S., et al. (2015) Characteristics and Outcomes for Patients with Advanced Vaginal or Vulvar Cancer Referred to a Phase I Clinical Trials Program: The MD Anderson Cancer Center Experience. Gynecologic Oncology Research and Practice, 2, 1. 
https://doi.org/10.1186/s40661-015-0018-x

[10] Woelber, L., Trillsch, F., Kock, L., Grimm, D., Petersen, C., Choschzick, M., et al. (2013) Management of Patients with Vulvar Cancer: A Perspective Review According to Tumour Stage. Therapeutic Advances in Medical Oncology, 5, 183-192. https://doi.org/10.1177/1758834012471699

[11] Fons, G., Hyde, S.E., Buist, M.R., Schilthuis, M.S., Grant, P., Burger, M.P., et al. (2009) Prognostic Value of Bilateral Positive Nodes in Squamous Cell Cancer of the Vulva. International Journal of Gynecological Cancer, 19, 1276-1280. https://doi.org/10.1111/IGC.0b013e31819d58a1

[12] Mahner, S., Prieske, K., Grimm, D., Trillsch, F., Prieske, S., von Amsberg, G., et al. (2015) Systemic Treatment of Vulvar Cancer. Expert Review of Anticancer Therapy, 15, 629-637. https://doi.org/10.1586/14737140.2015.1037837

[13] Xu, R., Jiang, W. and Guan, Z. (2011) Medical Oncology. People's Medical Publishing House, Beijing, Vol. 20, 16-17.

[14] Lim, S.H., Lee, J.Y., Sun, J.-M., Ahn, J.S., Park, K. and Ahn, M.-J. (2014) Comparison of Clinical Outcomes Following Gefitinib and Erlotinib Treatment in Non-Small-Cell Lung Cancer Patients Harboring an Epidermal Growth Factor Receptor Mutation in Either Exon 19 or 21. Journal of Thoracic Oncology, 9, 506-511. https://doi.org/10.1097/JTO.0000000000000095

[15] Bacha, O., Levesque, E., Renaud, M. and Lalancette, M. (2010) A Case of Recurrent Vulvar Carcinoma Treated with Erlotinib, an EGFR inhibitor. European Journal of Gynaecological Oncology, 32, 423-424.

[16] Richard, S., Krivak, T., Beriwal, S. and Zorn, K. (2008) Recurrent Metastatic Vulvar Carcinoma Treated with Cisplatin plus Cetuximab. International Journal of Gynecological Cancer, 18, 1132-1135. https://doi.org/10.1111/j.1525-1438.2007.01145.x

Submit or recommend next manuscript to OALib Journal and we will provide best service for you:

- Publication frequency: Monthly

- 9 subject areas of science, technology and medicine

- Fair and rigorous peer-review system

- Fast publication process

- Article promotion in various social networking sites (LinkedIn, Facebook, Twitter, etc.)

- Maximum dissemination of your research work

Submit Your Paper Online: Click Here to Submit

Or Contact service@oalib.com 